Review

\title{
Effect of Certain Disinfectants and Antibiotics on the Biofilm Formed by Staphylococcus Aureus Isolated from Medical Devices at the University Hospital Center of Sidi Bel Abbes (Algeria)
}

\author{
${ }^{1,2}$ Ibtissem Kara Terki, ${ }^{1}$ Hafida Hassaine, ${ }^{3}$ Assia Kara Terki, ${ }^{4}$ Bessouh Nadira, \\ ${ }^{1}$ Samia Bellifa, ${ }^{1}$ Imen Mhamedi and ${ }^{1}$ Meriem Lachachi \\ ${ }^{1}$ Laboratory of Food, Biomedical and Environnemental Microbiology (LAMAABE), \\ ${ }^{2}$ University of Djilali lyabes Sidi Bel Abbes, Algeria \\ ${ }^{3}$ University Abou Bekr Belkaid. Tlemcen \\ ${ }^{4}$ Ecole Supérieure de Management Tlemcen
}

Article history

Received: 03-04-2019

Revised: $10-05-2019$

Accepted: 12-09-2019

\section{Corresponding Author:}

Ibtissem Kara Terki

${ }^{1}$ Laboratory of Food,

Biomedical and

Environnemental Microbiology (LAMAABE).

${ }^{2}$ University of Djilali lyabes

Sidi Bel Abbes

Email: ibti.kara@gmail.com

\begin{abstract}
Staphylococcus aureus is one of the species that are most frequently isolated from medical devices. The ability to produce a biofilm is an important step in the pathogenesis of these staphylococci; biofilm formation is strongly dependent on the environmental conditions and also on the antibiotics and disinfectants used in the treatment of infections. In this study, 28 staphylococcus aureus isolated from medical devices at the University Hospital Center of Sidi Bel Abbes (in Northwestern Algeria) were analyzed to detect the formation of biofilm by culture on Red Congo Agar (RCA). The Tube Method (TM) and tissue Culture Plate (TCP) techniques were also used to investigate the effect of penicillin, ethanol and Betadine on the preformed biofilm. It has been found that 19 strains produced a bacterial slime on the Congo red medium, 7 strains produced a biofilm by the tube method, 2 of which are highly productive. In addition, 9 strains produced a biofilm on polystyrene micro-plates; this number was higher in the presence of penicillin and ethanol with 19 and 11 biofilm producing strains, respectively. On the other hand, no biofilm was formed in the presence of Betadine. It is important to examine the response of biofilms following an imposed external constraint such as disinfectants and antibiotics in order to develop new strategies to combat bacterial biofilms but also to better control their formation.
\end{abstract}

Keywords: Staphylococcus aureus, Biofilm, Medical Devices, Disinfectants, Antibiotics

\section{Introduction}

Staphylococcus aureus is one of the major causes of nosocomial and community-acquired infections. This germ is responsible for acute and chronic infections; most of them are due to the ability of these germs to adhere to medical implants and form a biofilm (Verma and Singh, 2015).

The biofilm is recognized as the most predominant form of development of bacteria in nature. These bacteria are complex communities of microorganisms, embedded in a self-secreted matrix of Extracellular Polymeric Substances (EPS) (Kara Terki et al., 2013).

Biofilms form on the surface of most materials, whether biological or not. According to the Center for Disease Control and Prevention (CDC), 65\% of bacterial infections are due to the presence of biofilms. In addition, infections associated with biofilms constitute a major clinical problem and are the cause of increased mortality; they generally require higher costs of medical treatment (Bellifa et al., 2016; Chessa et al., 2016; Badran et al., 2015). 
The development of biofilms depends strongly on the environmental conditions in which they are formed; it also depends on the different parameters these biofilms are subjected to. Any change in any of these parameters is usually perceived as stress and can trigger a particular response within the biofilm at any time (Ouchar Mahamat et al., 2013).

It is therefore essential to study the response of biofilms to an imposed external constraint, such as the action of disinfectants and antibiotics, in order to develop adequate strategies to fight biofilms but also to control and eventually limit their formation. The purpose of the present work fits within this particular context, which is to isolate Staphylococci from medical devices at some departments of the University Hospital Center of Sidi Bel Abbes for the purpose of evaluating the capacity of isolated strains to adhere to a surface and form a biofilm. This work aims also to investigate the influence of some disinfectants and antibiotics on the formation of staphylococcal biofilms.

\section{Materials and Methods}

\section{Bacterial strains}

The strains selected in this study were isolated from medical devices obtained from the Departments of Reanimation, Urology and Internal Medicine at the University Hospital Center (CHU) of Sidi Bel Abbes, a city located in northwestern Algeria.

\section{Identification}

After ablation of the devices, the microbiological analysis was carried out using the "Brun-Buisson" technique" (Brun-Buisson, 1994) which consists in rinsing the catheter lumen with saline solution and vortexing its intravascular end before culturing on a Chapman agar medium; this operation allows for the selection of the staphylococci.

Bacterial identification was performed by conventional methods, such as colony morphology, Gram stain, catalase production, coagulase assay and also by the API STAPH system (Biomérieux®).

\section{Detection of Biofilm Formation}

\section{Method of Red Congo Agar}

The Congo red test was performed as previously described by Freeman et al. (1989). The medium consisted of brain heart infusion broth (BHIB, $37 \mathrm{~g} / \mathrm{L})$, sucrose (50 $\mathrm{g} / \mathrm{L})$, agar no.1 (10 g/L) and Congo red stain $(0.8 \mathrm{~g} / \mathrm{L})$. Congo red was prepared as a concentrated aqueous solution and autoclaved at $121^{\circ} \mathrm{C}$ for $15 \mathrm{~min}$, separately from the other medium constituents and was then addedto the mixture when the agar had cooled to $55^{\circ} \mathrm{C}$. The plates were inoculated and incubated aerobically for 24 to $48 \mathrm{~h}$ at $37^{\circ} \mathrm{C}$. Biofilm producers form black colonies on CRA, whereas non-producers form red colonies. The Congo red dye directly interacts with certain polysaccharides, forming colored complexes (Jain and Agarwal, 2009).

\section{Tissue Culture Plate (TCP) Method}

Quantitative determination of biofilm formation in 96well microplates was performed according to Christensen et al. (1985) recommendations, but with changes the in incubation time which was extended to 48 hours. After culturing the bacterial strains in the BHIB medium and incubating for $18 \mathrm{~h}$ at $37^{\circ} \mathrm{C}$, the mixture is diluted $1 / 100$ in fresh (BHIB) medium. Then, the wells of a 96-well plate are filled with $0.2 \mathrm{ml}$ of this dilution and incubated at $37^{\circ} \mathrm{C}$. The microplate wells are then washed 3 times with distilled water, dried in the inverted position and stained with $0.5 \%$ (P.V) crystal violet solution.

The adherent cells are resuspended in $95 \%$ ethanol solution and the absorbance is measured at $540 \mathrm{~nm}$ using an Auto Reader ELISA reader (Model 680, Biorad, UK). The isolates are then classified into three categories as: (a) not-adhering, with an optical density less than 0.120 ; (b) weakly adhering, with an optical density greater than 0.120 or less than or equal to 0.240 and (c) strongly adhering, with an optical density greater than 0.240 .

\section{Tube Method}

This technique, developed in 1982 by Christensen et al. provides a qualitative assessment of the biofilm formation. From a young culture of $24 \mathrm{~h}$, a colony is sown in $10 \mathrm{~mL}$ of Brain Heart Infusion Broth (BHIB) supplemented with 2\% sucrose. After incubation at $37^{\circ} \mathrm{C}$ for $24 \mathrm{~h}$, the tubes are washed with Phosphate Buffered Saline (PBS) at $\mathrm{pH}=7.3$ and then dried. Each tube is then stained with crystal violet $(0.1 \%)$ for $5 \mathrm{~min}$. Once the dye is removed, the tubes are washed with distilled water and allowed to dry. The formation of the biofilm is considered positive when a visible film doubles the wall of the tube as well as its bottom. The formation of a ring at the liquid interface is not indicative of biofilm formation (Mathur et al., 2006).

\section{Effect of Certain Antiseptics and Antibiotics on Biofilm Formation using the TCP Technique}

\section{Antiseptics and Antibiotics under Study}

The main antiseptics used at the Hospital University Center of Sidi Bel abbes are polyvidone iodine (PVPI) and ethyl alcohol at $70^{\circ}$. As for antibiotics, penicillin $\mathrm{G}$ is by far the most widely used antibiotic in the hospital.

Polyvidone iodine is marketed under the name of Betadine ${ }^{\circledR} 10 \%$ (Laprophan laboratory). Also, the ethyl alcohol at $70^{\circ}$ is prepared at the laboratories of the hospital pharmacy of the University Hospital by diluting the alcohol at $90^{\circ}$ and penicillin $\mathrm{G}$ ( 1 million); it is marketed by SAIDAL laboratories. 


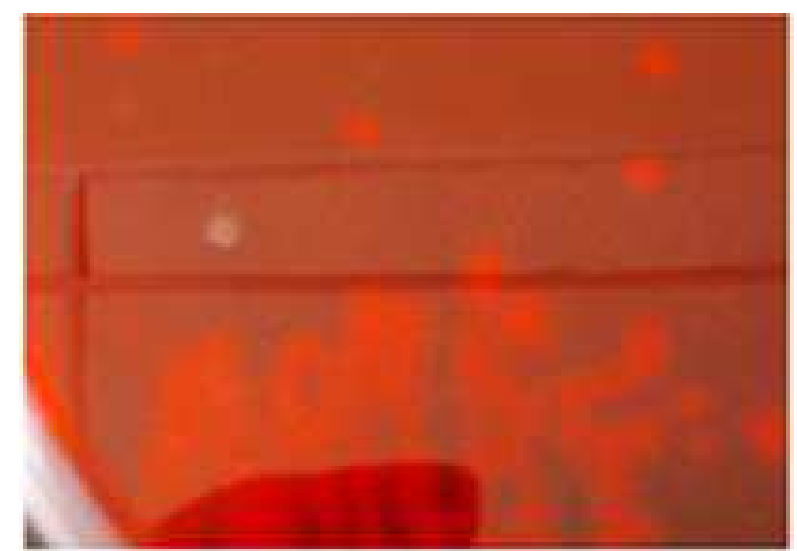

(A)

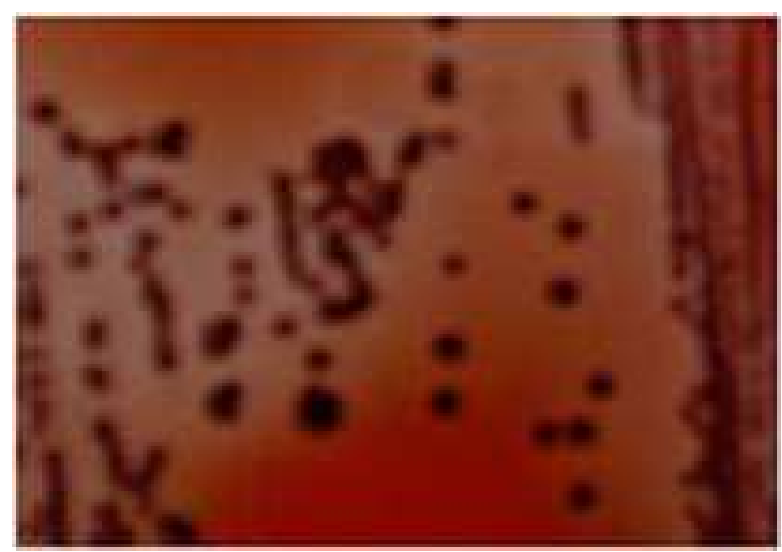

(B)

Fig. 1: CRA plate test (A): Non slime producing strains / (B): slime-producing strains

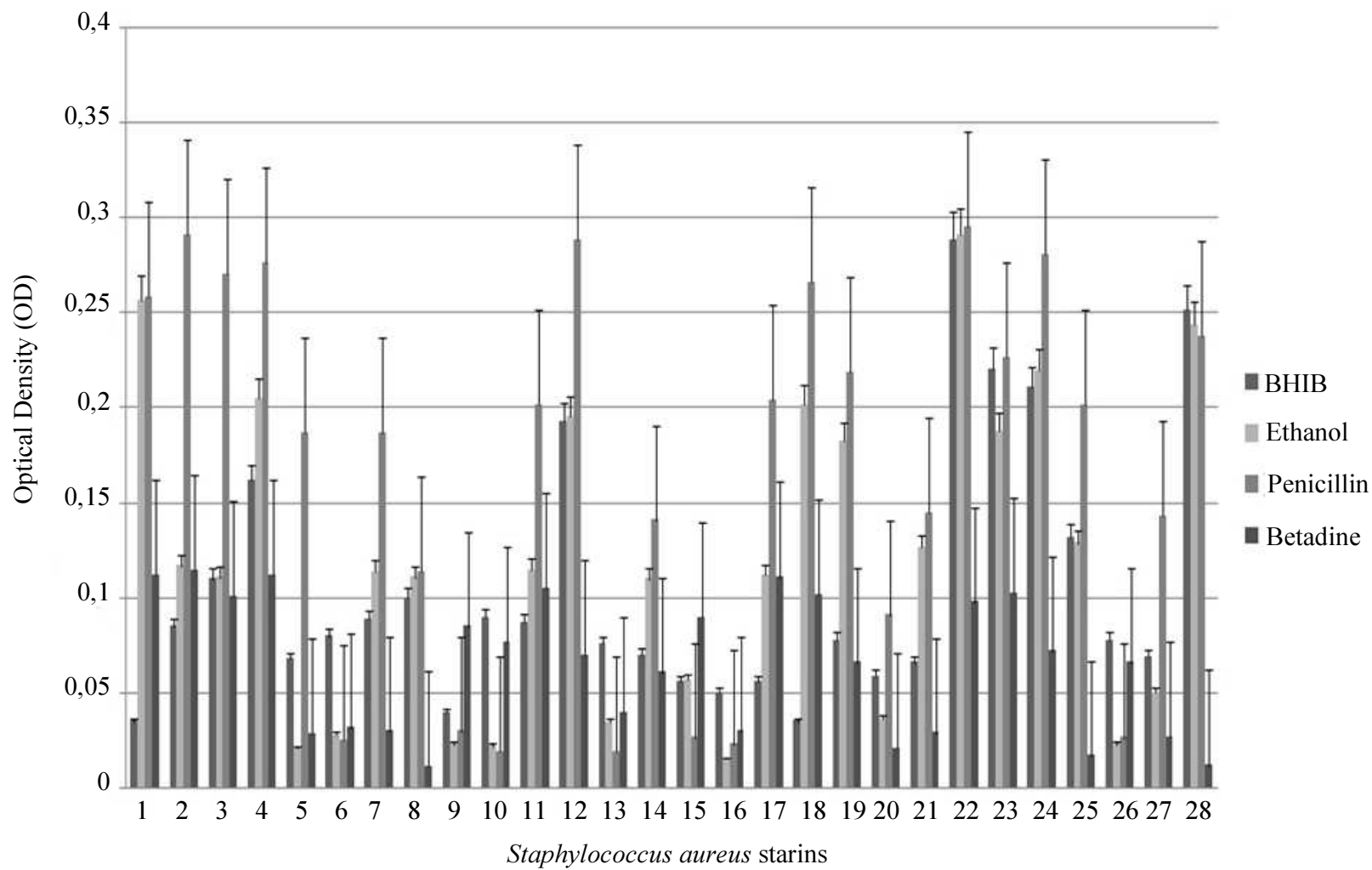

Fig. 2: Biofilm formation of Staphylococcus aureus strains on BHIB, ethanol 70\%, Penicillin and betadine $10 \%$. Adherent bacterial biofilms were stained with Crystal violet as described in Materials and methods. A strain was considered biofilm-positive, if its OD was higher or equal to $0.120, \mathrm{P}^{*}<0: 05$ (t-test). Data are representative of 3 replicate experiments

\section{Biofilm Assay}

After forming a 48-hr young biofilm by the TCP technique (previously described), the 96-well microplate are rinsed 3 times with distilled water and dried. Then, Penicillin G (1million U.I), Betadine $10 \%$ (an iodinated derivative) and ethyl alcohol $70^{\circ}$ are added to the biofilm. The microplate is then formed and incubated for $24 \mathrm{~h}$.

After incubation, the wells of the microplate are carefully rinsed, dried and stained with crystal violet according to the standard technique. The Optical Density (OD) is measured at $490 \mathrm{~nm}$ by the ELISA reader. 
Table 1: Results of biofilm formation by Staphylococcus aureus isolated from medical devices

\begin{tabular}{|c|c|c|c|c|c|c|c|c|}
\hline \multirow[b]{2}{*}{ Strain } & \multirow[b]{2}{*}{ Unit } & \multirow[b]{2}{*}{ Medical device } & \multicolumn{4}{|l|}{ TCP } & \multirow[b]{2}{*}{$\mathrm{TM}$} & \multirow[b]{2}{*}{ Production of slime } \\
\hline & & & BHIB & Ethanol & penicilin & betadine & & \\
\hline$\overline{\text { S1 }}$ & Urology & U.C & - & ++ & ++ & - & - & + \\
\hline $\mathrm{S} 2$ & Urology & U.C & - & - & ++ & - & - & + \\
\hline S3 & Urology & U.C & - & - & ++ & - & - & + \\
\hline S4 & Urology & U.C & + & + & ++ & - & + & + \\
\hline S5 & Urology & U.C & - & - & + & - & + & + \\
\hline S6 & Urology & U.C & - & - & - & - & - & + \\
\hline S7 & Urology & U.C & - & - & + & - & - & + \\
\hline S8 & Urology & U.C & - & - & - & - & - & - \\
\hline S9 & Urology & U.C & - & - & - & - & - & - \\
\hline S10 & Urology & U.C & - & - & - & - & - & - \\
\hline S11 & Urology & U.C & - & - & + & - & - & - \\
\hline S12 & Urology & U.C & + & + & ++ & - & + & + \\
\hline S13 & Urology & U.C & - & - & - & - & - & - \\
\hline S14 & intensive care & C.V.C & - & - & + & - & - & - \\
\hline S15 & intensive care & C.V.C & - & - & - & - & - & - \\
\hline S16 & intensive care & C.V.C & - & - & - & - & - & - \\
\hline S17 & intensive care & C.V.C & - & - & + & - & - & + \\
\hline S18 & intensive care & C.V.C & - & + & ++ & - & + & + \\
\hline S19 & intensive care & C.V.C & - & + & ++ & - & + & + \\
\hline $\mathrm{S} 20$ & intensive care & C.V.C & - & - & - & - & - & + \\
\hline S21 & intensive care & C.V.C & - & + & + & - & - & + \\
\hline S22 & intensive care & C.V.C & ++ & ++ & + & - & ++ & + \\
\hline S23 & Internal Medicine & U.C & + & + & + & - & - & + \\
\hline S24 & Internal Medicine & U.C & + & + & ++ & - & - & + \\
\hline S25 & Internal Medicine & U.C & + & + & + & - & - & + \\
\hline S26 & Internal Medicine & C.V.C & - & - & - & - & - & + \\
\hline S27 & Internal Medicine & C.V.C & - & - & + & - & - & + \\
\hline S28 & Internal Medicine & C.V.C & ++ & ++ & + & - & ++ & + \\
\hline
\end{tabular}

C.V.C: central venous catheters, U.C: urinary catheters, (++): biofilm forsmation good, (+): biofilm formation average, (-) :non biofilm formation

\section{Results}

\section{Samples}

A total of 42 samples were taken at the Sidi Bel Abbes Teaching Hospital; a number of 28 strains were retained, after biochemical identification using the API 20 staph gallery and the coagulase test. All the strains were identified as staphylococcus aureus.

\section{Results of the Red Congo Agar Technique}

The search for slime production on Congo red medium revealed that 19 out of 28 strains, isolated from medical devices, are slime-producing and show black colonies with a dry-consistency crystalline on the Congo Red Agar. This aspect is due to the production of exo-polysaccharides that react with Congo red, while 09 strains were non-slime producing and showed red colonies on the same medium (Fig. 1).

\section{Results of the Tissue Culture Plate Technique}

Quantitative determination of biofilm formation within all our strains was performed by microplate technique (TCP), as shown in Fig. 2.
Using the BHIB growth medium, only 9 strains produced biofilm; 7 of them are low producing and 2 high producing of biofilms (Table 1).

\section{Results of the Tube Method}

The results obtained by the Tube Method (Table 1) are different from those obtained by the TCP technique; indeed, only 7 strains produce biofilms by this technique, 2 of which are highly productive.

\section{Effect of Ethanol, Penicillin and Betadine on the Biofilm}

For the purpose of assessing the impact of environmental conditions on the staphylococcus biofilm, the three substances were added after a period of $48 \mathrm{~h}$. This has led to an increase in the number of strains capable of forming a biofilm by the TCP technique; indeed, 11 strains produced a biofilm in the presence of ethanol and 19 strains gave a biofilm in the presence of penicillin which appears to stimulate greater biofilm formation. Indeed, 8 strains are found to be good biofilm producers and 11 are moderate biofilm producers. Moreover, only 3 strains are highly biofilm producers 
and 11 are moderate producers in the presence of ethanol (Table land Fig. 2).

On the other hand, no strain could form a biofilm in the presence of Betadine.

\section{Discussion}

Staphylococcus aureus is one of the most common germs that are responsible for foreign body infections of central venous catheters, mechanical heart valves and urinary catheters. Their major virulence factors are the ability to produce an extracellular matrix and form a biofilm; this makes the clinical treatment extremely difficult (Derek et al., 2017).

Early detection of staphylococcal biofilms may be one of the essential steps for the prevention and treatment of infection on medical devices (Martín-López et al., 2002).

The present article aims mainly to evaluate the formation of biofilms by staphylococcus aureus isolated from medical devices at the Hospital University Center of Sidi Bel Abbes using RCA, TM and TCP techniques.

The results obtained reveal that 19 out of 28 strains can produce slime by culture on Congo Red medium. These results turn out to be in good agreement with those found by Arciola et al. (2001) who reported significant slime production in staphylococcus aureus $(60.8 \%)$.

The production of slime is revealed by the appearance of black colonies on Congo Red medium (Fig.1). This aspect is mainly due to the production of Polysaccharide Intercellular Adhesin (PIA) that reacts with the culture medium. Described for the first time in Staphylococcus epidermidis, by Mack et al. (1992), the PIA is generally located on the surface of the cell. It is coded by the locus ica and plays an important role in intercellular adhesion; as a result, PIA has been recognized as a crucial factor in the colonization of medical equipment by staphylococci (Cramton et al., 2001; Martín-López et al., 2002; Derek et al., 2017).

The results obtained by the TCP technique reveal that only 9 out of 28 strains can produce a biofilm in the BHIB medium. These results are in agreement with the observations of other authors who have shown that few strains are biofilm-forming in a growth medium without supplement (sugar, antibiotics or NACL) (Cho et al., 2002; Johannes et al., 2002; Mathur et al., 2006).

In our study, it was found that 7 of the 28 strains could produce a biofilm by the Tube technique. Also, a good correlation was noted between the Tissue Culture Plate (TCP) method and the Tube Method (TM) for the high biofilm producing strains (Table 1). It is worth noting that a large variability in the positive and negative biofilm classification was observed by the Tube Method (TM). Similarly, it was difficult to differentiate between biofilm low-producing and non-producing strains. Therefore, the present work allows us to confirm the hypothesis already formulated by several authors, including Mathur et al. (2006), according to which the Tube Method (TM) cannot routinely be recommended.

Consequently, the TCP technique should be more widely utilized as it is considered as a standard test for the detection of biofilm formation. This method has been recognized as being the most sensitive, accurate and reproducible for the determination of staphylococcal biofilm formation. It allows for a quantitative assessment in order to compare the adhesion of different strains and also to examine a large number of isolates simultaneously (Racha et al., 2012).

Furthermore, it is a quantitative, practical and economical technique that allows for the identification of optimal culture factors and conditions for biofilm formation (Castro Melo et al., 2013).

Indeed, biofilm formation is a complex phenomenon, which can be affected by many factors, particularly the surrounding environment (Branger et al., 2007). In order to study the influence of certain factors of the surrounding environment at the Hospital University Center of Sidi Bel Abbes on the biofilm of Staphylococcus aureus, it was decided to use the TCP technique described above. It was noted that after the addition of penicillin and ethanol, the number of biofilm-forming strains increased to 11 in the presence of ethanol and to 19 in the presence of penicillin. These observations are consistent with those made in other studies that suggest a strong dependence between growth conditions and biofilm formation in staphylococci (Mathur et al., 2006; Kevin et al., 2016).

Luther et al. (2015; Redelman et al., 2012) showed that ethanol encourages biofilm formation in all strains studied. Similarly, El-Banna et al. (2010) showed that antibiotics also promote biofilm formation in staphylococci isolated from medical devices at the University Hospital Center of the City of Alexandria (Egypt).

Based on our 2013 results (Kara Terki et al., 2013) and those found in the literature, it has been found that the $i c a$ operon expression, which is responsible for the production of extracellular polysaccharides in staphylococcus aureus, depends on the environmental conditions, such as growth media composition, temperature, osmolarity, the presence of oxygen and sub-inhibitory concentrations of antibiotics; it was also revealed that large concentrations of $\mathrm{NaCl}$ increase biofilm formation and strongly induce ica expression in staphylococci. The presence of divalent cations, such as calcium and magnesium, increases the production of polysaccharides, which leads to an amplification of biofilm formation. In staphylococci, the expression of the ica ADBC genes can also be influenced by other environmental conditions, such as oleic acid and iron limitation (Gotz, 2002; Chaieb et al., 2005; Derek et al., 2017).

On the other hand, the resistance of biofilm-included bacteria to antibiotics and disinfectants is well acknowledged. According to some authors, this resistance is attributed to certain factors, such as the 
bacteria physiology, the power of matrices and many others (Olsen, 2015). Scientific work on the subject shows that there is a strong link, direct or indirect, between the architecture of the biological structure and its resistance to antimicrobial action (Abdel Halim et al., 2018). Indeed, the multiplication of adherent cells and the production of an extracellular matrix lead to the development of a complex structure in which the biocides and antibiotics can meet diffusion problems, which limits their effectiveness. This structure controls the establishment of nutrient, oxygen and metabolite product concentration gradients, resulting in chemical and nutritional heterogeneity within the biofilm (Stewart, 2015). In response to their local microenvironment, the cells can then evolve into tolerance phenotypes by physiological changes and/or by the expression of specific genes. Therefore, the overall resistance of the bacterial community appears to be a multi-factorial structural-dependent process that involves local phenomena. Several factors may explain this strong resistance. The polymeric matrix, which acts as a barrier to reduce or prevent the diffusion of antimicrobial agents and the low concentration gradients of oxygen and certain nutrients, cause some cells in the biofilm to be metabolically inactive and may even remain in dormant form. These dormant bacterial cells are probably responsible for much of the tolerance associated with biofilms (Wojtyczka et al., 2014; Thien-Fah et al., 2001). Repeated exposure to disinfectant and antibiotics concentrations can generate some physiological adaptations that further delay the subsequent tolerance of the biofilm. When a community of adherent bacterial cells is subject to antibiotics and disinfectants, only a few are able to resist (Amiyare et al., 2015). On the other hand, in the presence of Betadine (polyvidone iodine), the optical density decreases in all the strains and none of them is able to form a biofilm.

The results of Essayagh et al. (2010) confirm ours which reveal that PVPI is the best of antiseptics studied. In fact, only six strains (4.6\%) out of the 130 tested, could resist to the PVPI that was available at the pharmacy, while $40(30.7 \%)$ were resistant to iodinated alcohol and 20 (15.4 $\%$ ) to alcohol at $70^{\circ} \mathrm{C}$. Chemical analysis also confirms this finding. Indeed, PVPI is a stable molecule consisting of an iodine complex and a water soluble organic agent that slowly transports and releases iodine. This structure makes the PVPI less irritating, less allergenic and more stable over time; while iodized alcohol and ethyl alcohol at $70^{\circ} \mathrm{C}$ become stable after fifteen days and one month, respectively, after the date of their preparation (Clevenot et al., 2003; Vaillant, 2005).

\section{Conclusion}

Although a lot of research has been carried out in the field of biofilms, current treatments for their development are still limited. However, recent findings from basic research have identified mechanisms or regulatory pathways that represent potential therapeutic approaches. However, it is likely that biofilms develop some strategies to resist to anti-infective agents and disinfectants, which requires new adaptive, elaborate and systematic plans of action to combat them.

\section{References}

Abdel Halim, R.M., N.N. Kassem and B.S. Mahmoud, 2018. Detection of biofilm producing staphylococci among different clinical isolates and its relation to methicillin susceptibility. Macedonian J. Med. Sci., 20: 1335-1341. DOI: 10.3889/oamjms.2018.246

Amiyare, R., A. Esmail, Y. Ghanmi and M. Ouhssine, 2015. Evaluation de L'effet d'un désinfectant à base de Glutaraldehydeà $2 \%$ sur le biofilm d'Acinetobacter baumannii. J. Mater. Environ. Sci., 6: 3168-3173.

Arciola, C.R., L. Baldassarri and L. Montanaro, 2001. Presence of icaA and icaD genes and slime production in a collection of staphylococcal strains from catheter-associated infections. J. Clin. Microbiol., 39: 2151-2156.

DOI: 10.1128/JCM.39.6.2151-2156.2001

Badran, H., M. Salah, M. Fawzy, A. Sayed and D. Ghaith, 2015. Detection of bacterial biofilms in chronic pharyngitis resistant to medical treatment. Ann. Otol. Rhinol. Laryngol., 124: 567-71. DOI: $10.1177 / 0003489415570934$.

Bellifa, S., H. Hassaine, I. Kara Terki and W. Didi et al., 2016. Study of biofilm production and antimicrobial resistance pattern of Klebsiella pneumoniae isolated from urinary catheter at the university hospital of Tlemcen. Am. J. Microbiol. Biotechnol., 3: 13-17.

Branger, A., M.M. Richer and S. Roustel, 2007. Some microbial system: biofilms. In: Microbiochemistry and food. Educagri Edition, Dijon, pp: 131-164.

Brun-Buisson, C., 1994. Critical Analysis of Diagnostic Methods of Catheter-Related Infection on Removed Equipment. Réan Urg., 3: 343-6.

Castro Melo, P., L.M. Ferreira, A.N. Filho, L.F. Zafalon and H.I. Godoy Vicente et al., 2013. Comparison of methods for the detection of biofilm formation by Staphylococcus aureus isolated from bovine subclinical mastitis. Brazilian J. Microbiol., 44: 15178382. DOI: $10.1590 / \mathrm{S} 1517-83822013005000031$

Chaieb, K., K. Mahdouania and A. Bakhrouf, 2005. Detection of icaA and icaD loci by polymerase chain reaction and biofilm formation by Staphylococcus epidermidis isolated from dialysate and needles in a dialysis unit. J. Hospital Infec., 61: 225-230. DOI: 10.1016/j.jhin.2005.05.014 
Chessa, D., G. Ganau, L. Spiga, A. Bulla and V. Mazzarello et al., 2016. Staphylococcus aureus and Staphylococcus epidermidis virulence strains as causative agents of persistent infections in breast implants. PLOS ONE, 11: e0146668-e0146668. DOI: 10.1371/journal.pone.0146668

Cho, S., K. Naber, J. Hacker and W. Ziebuhr, 2002. Detection of the ica ADBC gene cluster and biofilm formation in Staphylococcus epidermidis isolates from catheter-related urinary tract infections. Int. J. Antimicrobial Agent., 19: 570-575. DOI: $10.1016 / \mathrm{S} 0924-8579(02) 00101-2$

Christensen, G.D., W.A. Simpson, J.J. Yonger, L.M. Baddor and F.F. Barrett et al., 1985. Adherence of coagulase-negative staphylococci to plastic tissue culture plates: A quantitative model for the adherence of staphylococci to medical devices. J. Clin Microbiol., 22: 996-1006. PMC271866

Clevenot, D., S. Robert, B. Debaene and O. Mimoz, 2003. Analyse critique de la littérature sur l'utilisation comparée de deux antiseptiques lors du cathétérisme vasculaire ou rachidien. Ann Fr Anesth., 22: 787-97.

Cramton, S.E., M. Ulrich and F. Gotz, 2001. Anaerobic conditions induce expression of polysaccharide intercellular adhesin in Staphylococcus aureus and Staphylococcus epidermidis. Infection Immunol., 69: 4079-4085. DOI:10.1128/IAI.69.6.4079-4085.2001

Derek, E., K. Moormeier and w. Bayles, 2017. Staphylococcus aureus, biofilm: A complex developmental organism. Molecular Microbiol., 104: 365-376. DOI: $10.1111 / \mathrm{mmi} .13634$

El-Banna, T., A. Abd El-Aziz, A. Abo-Kamar, A. Ghazal and R. Abo Zahra, 2010. In vitro activities of three kinds of antibiotics against Staphylococcal biofilm and planktonic cultures. African J. Microbiol. Res., 4: 2275-2282.

Essayagh, T., A. Elameri, A. Zohoun, M. Miloudi and S. Elhamzaoui, 2010. Activité antibactérienne des antiseptiques utilisés à l'Hôpital militaire d'instruction Mohamed V de Rabat. Ann. Biol. Clin., 68: 421-7. DOI: 10.1684/abc.2010.0447

Freeman, D.J., F.R. Falkner and C.T. Keane, 1989. New method for detecting slime production by coagulasenegative staphylococci. J. Clin. Pathol., 42: 872-874. DOI: $10.1136 /$ JCP.42.8.872

Gotz, F., 2002. Staphylococcus and biofilms. Molecular Microbiol., 43: 1367-1378.

DOI: $10.1046 / \mathrm{J} .1365-2958.2002 .02827 . X$

Jain, A. and A. Agarwal, 2009. Biofilm production, a marker of pathogenic potential of colonizing and commensal staphylococci. J. Microbiol. Methods, 76: 88-92: DOI: 10.1016/j.mimet.2008.09.017

Johannes, K.M., M.A. Knobloch, H. Horstkotte and M. Dietrich, 2002. Evaluation of different detection methods of biofilm formation in Staphylococcus aureus. Med. Microbiol. Immunol., 191: 101-106. DOI: 10.1016/j.mimet.2008.09.017
Kara Terki, I., H. Hassaine, S. Oufrid, S. Bellifa and I. Mhamedi et al., 2013. Detection of ICAA and ICA $\mathrm{D}$ genes and biofilm formation in Staphylococcus spp. isolated from urinary catheters at the University Hospital of TLEMCEN (Algeria). African J. Microbiol. Res., 7: 5350-5357. DOI: $10.5897 / J M R 2013.5873$

Kevin, D., A. Mlynek, T. Mary, A. Callahan and V. Anton et al., 2016. Effects of Low-Dose Amoxicillin on Staphylococcus aureus USA300 Biofilms. Antimicrobial Agents Chemotherapy., 60: 2639-2651. DOI: 10.1128/AAC.02070-15

Luther, M.K., S. Bilida, A. Leonard, 1. Merme and L. Kerry, 2015. Ethanol and isopropyl alcohol exposure increases biofilm formation in staphylococcus aureus and staphylococcus epidermidis. Infect Dis Ther., 4: 219-226. DOI: $10.1007 / \mathrm{s} 40121-015-0065-\mathrm{y}$

Mack, D., N. Siemssen and R. Laufs, 1992. Parallel induction by glucose of adherence and a polysaccharide antigen specific for plastic-adherent Staphylococcus epidermidis: Evidence for functional relation to intercellular adhesion. Infect. Immunol., 60: 2048-2057.

Martín-López, J.V., E. Pérez-Roth, F.C.M.O. Díez Gil, N. Batista and M. Morales et al., 2002. Detection of Staphylococcus aureus clinical isolates harboring the ICA Gene cluster needed for biofilm establishment. J. Clinical Microbiol., 40: 1569-1570. DOI: 10.1128/JCM.40.4.1569-1570.2002

Mathur, T., S. Singhal, S. Khan, D.J. Upadhyay and T. Fatma et al., 2006. Detection of biofilm formation among the clinical isolates of Staphylococci: An evaluation of three different screening methods. Indian J. Med. Microbiol., 24: 25-29. DOI: $10.4103 / 0255-0857.19890$

Olsen, I., 2015. Biofilm-specific antibiotic tolerance and resistance. Eur. J. Clin. Microbiol. Infect Dis., 34: 877-886. DOI: 10.1007/s10096-015-2323-z

Ouchar Mahamat, O., A. Tidjani, A. Savadogo, A. Abakabir Mahamat and M.K. Somda et al., 2013. Isolation and characterization of bacteria producing biofilms from food products. Rev. Microbiol. Ind. San et Environn., 7: 187-210.

Racha, A.N., H.M. Abu Shady and H.S. Hussein, 2012. Biofilm formation and presence of ICAAD gene in clinical isolates of staphylococci. Egyptian J. Medica. Human Genetic, 13: 269-274.

DOI: 10.1016/J.EJMHG.2012.04.007

Redelman, C.V., C. Maduakolam, G. Gregory and Anderson, 2012. Alcohol treatment enhances Staphylococcus aureus biofilm Development. FEMS Immunol. Med. Microbiol., 66: 411-418. DOI: $10.1111 / 1574-695 x .12005$ 
Stewart, P., 2015. Antimicrobial tolerance in biofilms. Microbiol. Spectr., 3: 10.1128 .

DOI: 10.1128/microbiolspec.MB-0010-2014

Thien-Fah, C., M. George and A. O'Toole, 2001. Mechanisms of biofilm resistance to antimicrobial agents. TRENDS Microbiol., 9: 1-1.

DOI: $10.1016 / \mathrm{S} 0966-842 \mathrm{X}(00) 01913-2$

Vaillant, L., 2005. Les antiseptiques, ce n'est pas automatique. Annal Dermatol., 132: 942-52.

Verma, P. and S. Singh, 2015. Clinical distribution of biofilm forming Staphylococcus aureus and its sensitivity against some antibiotics. World J. Pharmacy Pharmaceutical Sci., 4: 942-9520.
Wojtyczka, R., K. Orlewska, M. Kępa, D. Idzik and A. Dziedzi et al., 2014. Biofilm formation and antimicrobial susceptibility of Staphylococcus epidermidis strains from a hospital environment. Int. J. Environ. Res. Public Health, 11: 4619-4633. DOI: $10.3390 /$ ijerph110504619 